- Abstract -

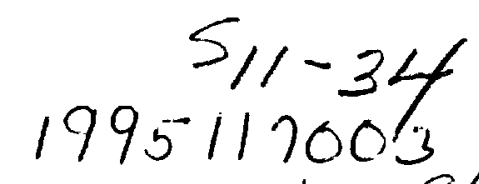

Workshop for Computational Fluid Dynamic Applications In Rocket Propulsion

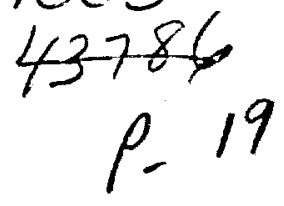

\title{
Three-Dimensional Navier-Stokes Analysis and Redesign of an Imbedded Bellmouth Nozzle in a Turbine Cascade Inlet Section
}

\author{
P. W. Giel, \\ J. R. Sirbaugh \\ Sverdrup Technology, Inc. \\ NASA LeRC Group \\ 2001 Aerospace Parkway \\ Brook Park, $\mathrm{OH} 44142$
}

(216) $826-6686$

Verification of proposed turbopump blading performance will involve evaluation of candidate blades in cascade test facilities. It is necessary to be able to predict the flow fields within these cascades for the results to be applicable to actual engine

environments. This work presents the results of a study to predict the flow field for the NASA Lewis Transonic Turbine Blade Cascade Facility, which is similar to those used to evaluate rocket propulsion turbines. A pitchwise non-uniform total pressure distribution was observed at the blade row leading edge plane. A CFD analysis was used to show that the cause of the flow non-uniformity was a pair of vortices that originated in an embedded bellmouth inlet. Further CFD analysis was used to verify that a redesigned inlet section resulted in a flow with acceptable uniformity.

A computational analysis was chosen because physical accessibility to the inlet section was limited, and because a computational approach also allows one to examine design changes cheaper and more quickly than an experimental approach would. The PARC code, a general purpose, three-dimensional, Navier-Stokes code with multi-block solution capability, was chosen for the present study. Results are presented detailing the computational requirements needed to accurately predict flows of this nature.

Calculations of the original geometry showed total pressure loss regions consistent in strength and in location to experimental measurements. An examination of the results shows that the distortions are caused by a pair of vortices that originate as a result of the interaction of the flow with the imbedded bellmouth. Computations were performed for an inlet geometry which eliminated the imbedded bellmouth by bridging the region between it and the upstream wall. This analysis indicated that eliminating the imbedded bellmouth eliminates the troublesome pair of vortices, resulting in a flow with much greater pitchwise uniformity. 


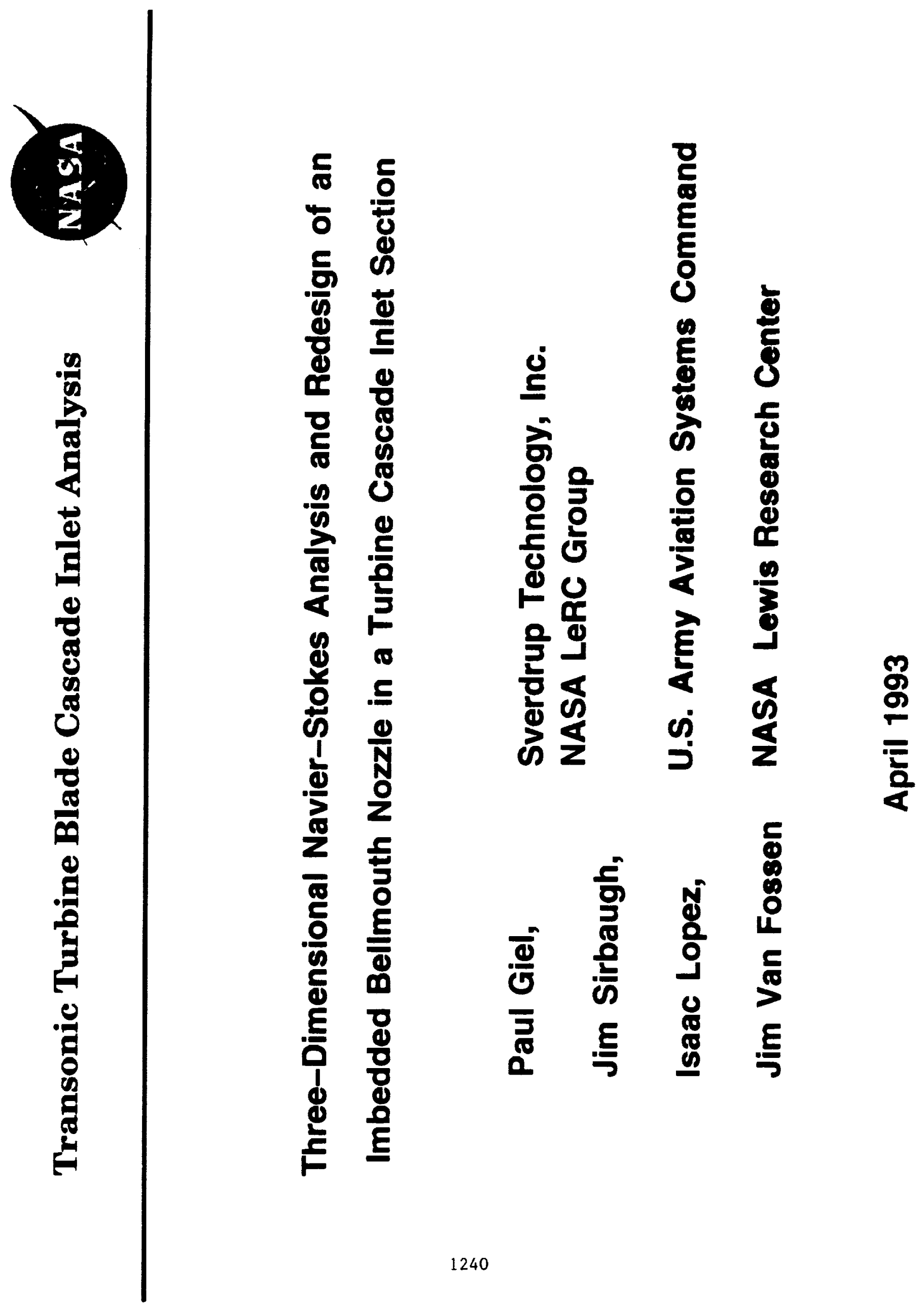



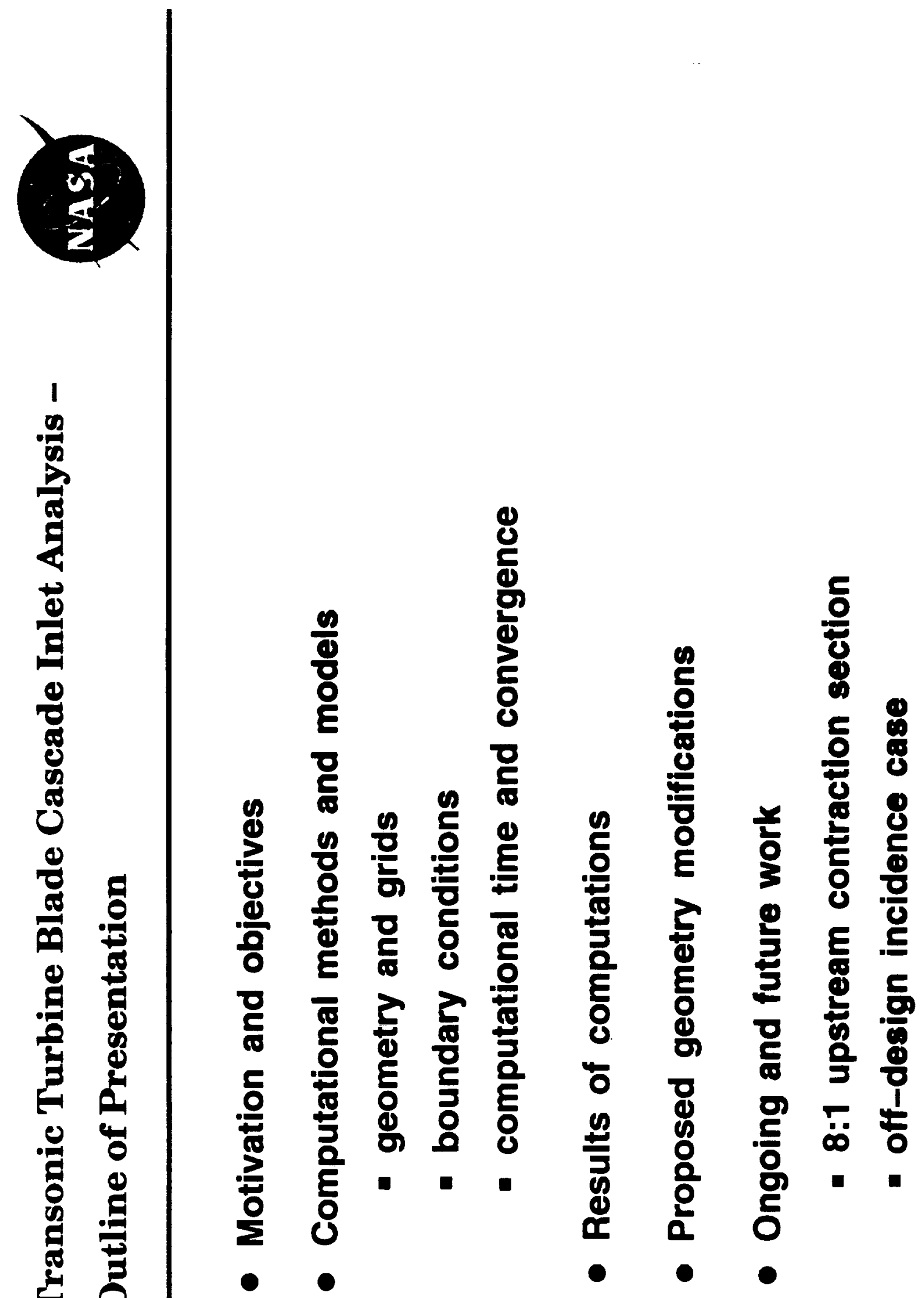


\section{$\left(\begin{array}{l}4 \\ 4 \\ 2 \\ 2\end{array}\right.$}
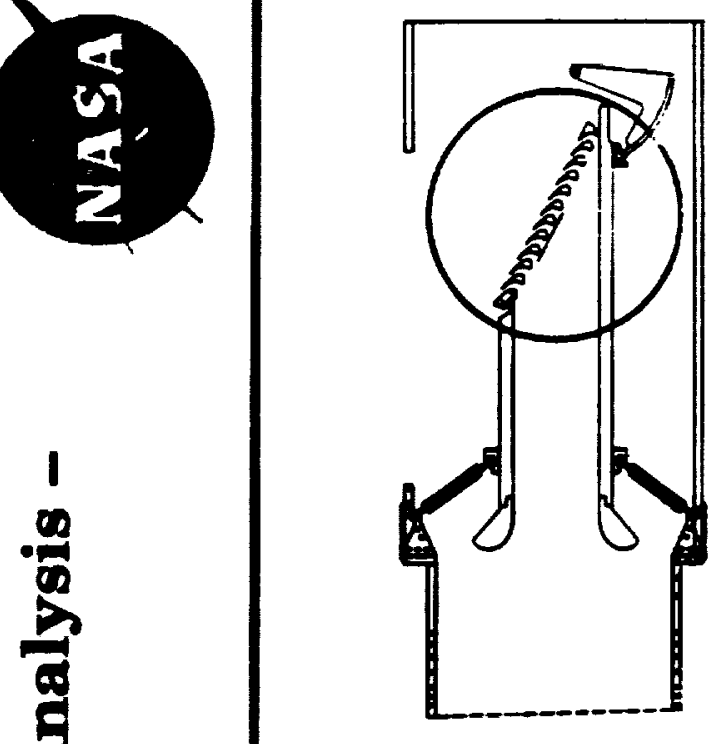

0
0
8
0
0
0
0
5
0
0
0
0
0
0
0
0
0
0
0
0
0
0

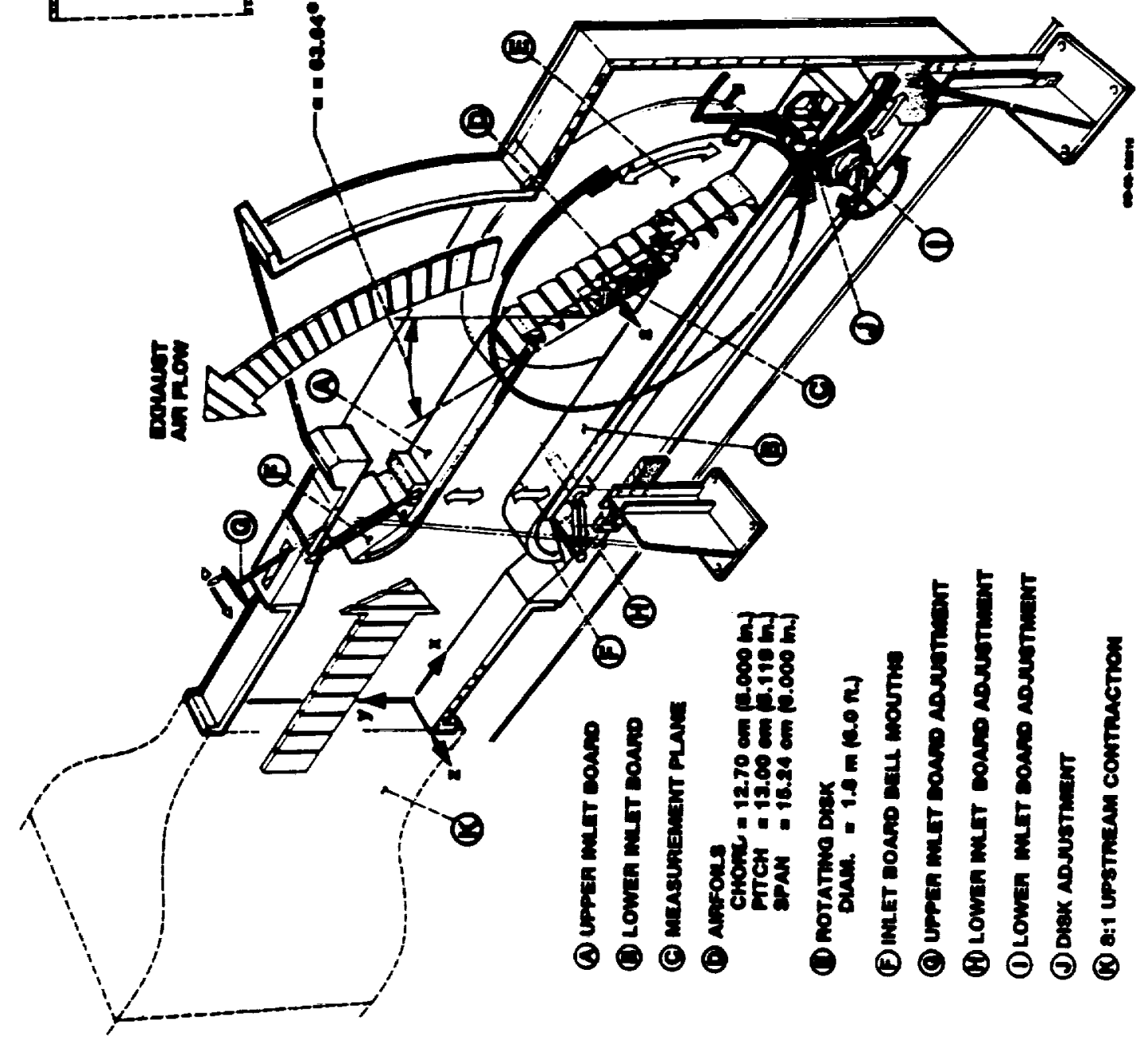



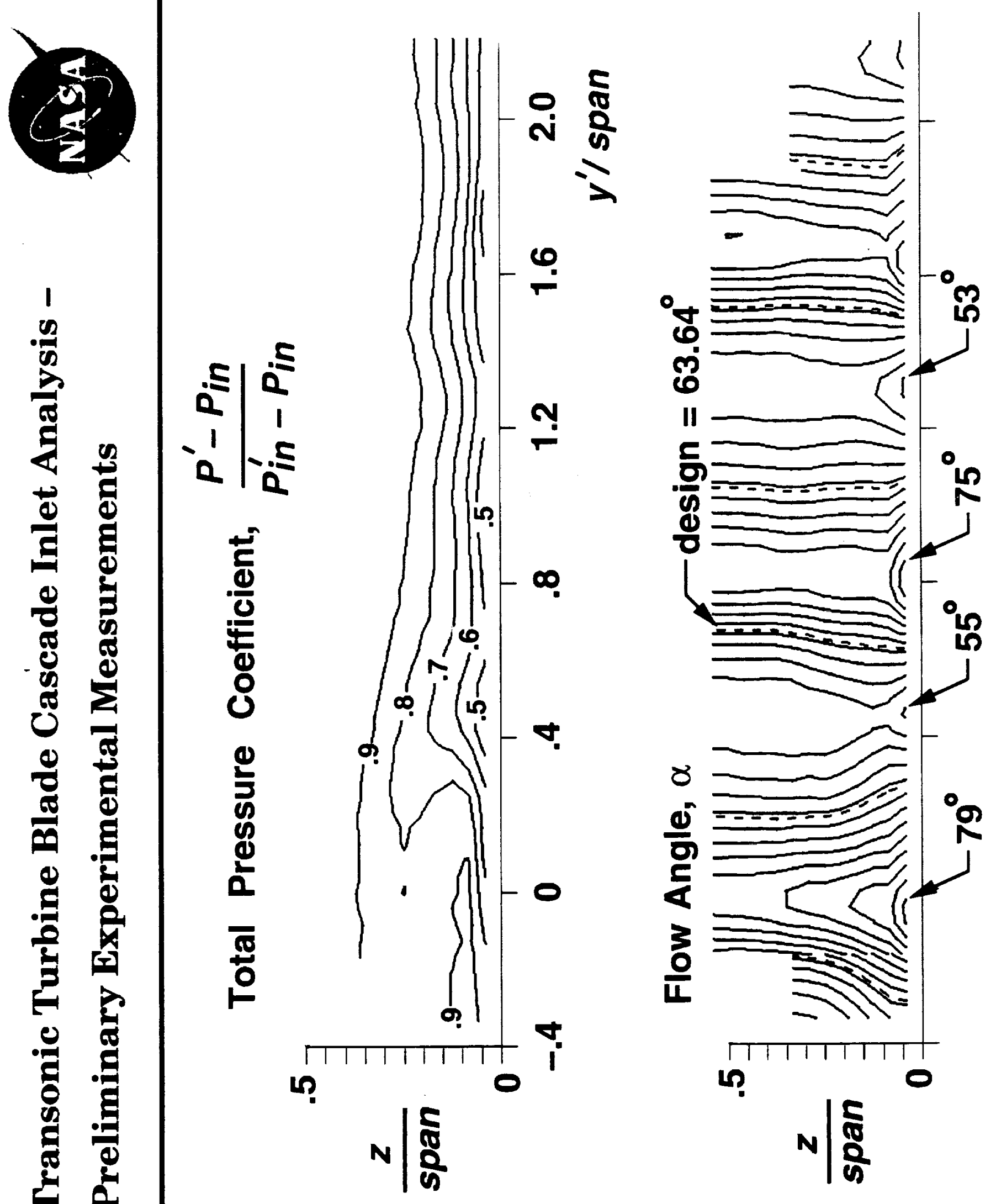


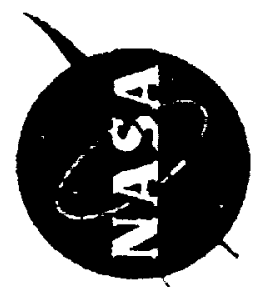

1
0
0
0
0
0
0
0
0
0
0
0
0
0
0
0
0
0
0
0
0

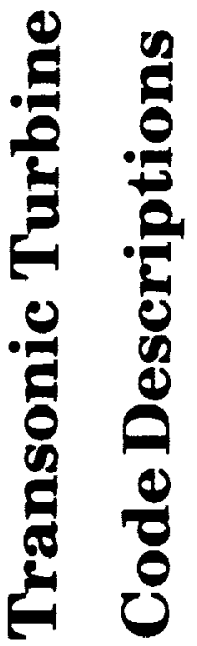

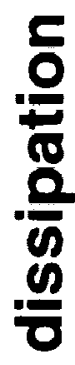

$\frac{1}{0}$

के

1

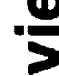

$\pi$

8

$\Phi$

ง

눙

웅 웅 응 동

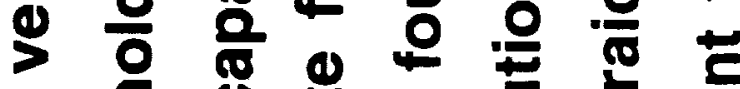

n 50 잉 0 응

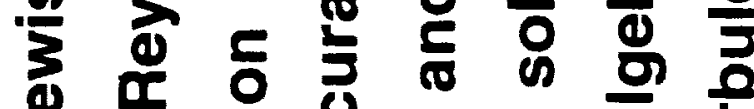

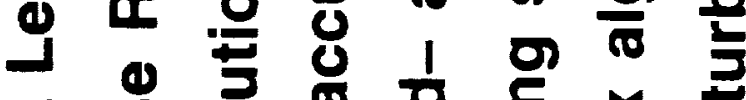

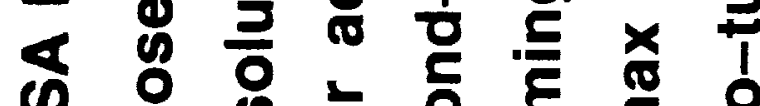

운

ล

훙

8 응 응 $\frac{5}{0}$

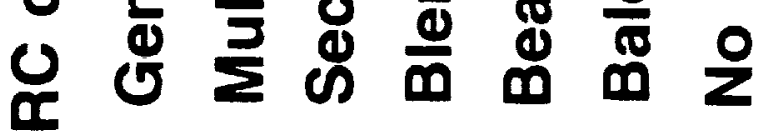

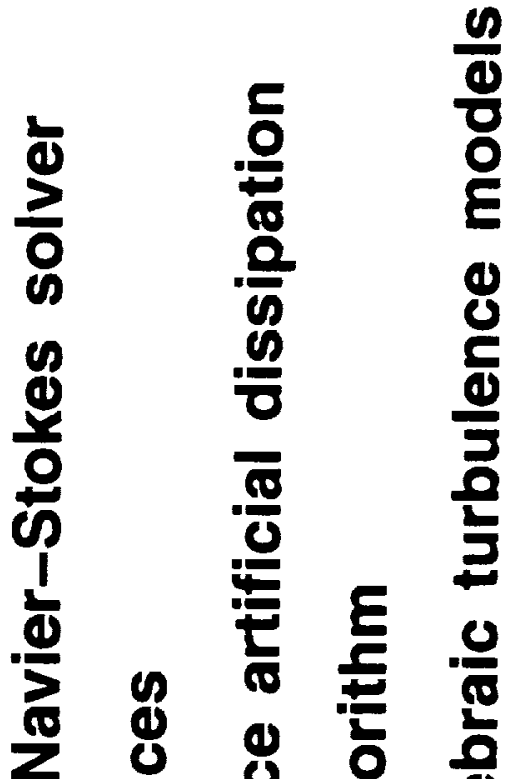

2

잉 $\frac{6}{6}$

\%

잉 훙 흥 을 틀

$\geqslant \pm 1$ 응

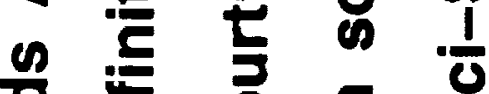

우요

요용

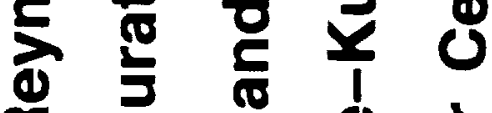

○ 8 1

난

ㅎํㅇㅇํ

틀 응

उ 0 क क 1

ह $\frac{1}{0}$ व $\frac{\pi}{5}$

응 ㅇํำ

은 8 응

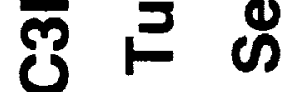

$\boldsymbol{m}$

u 

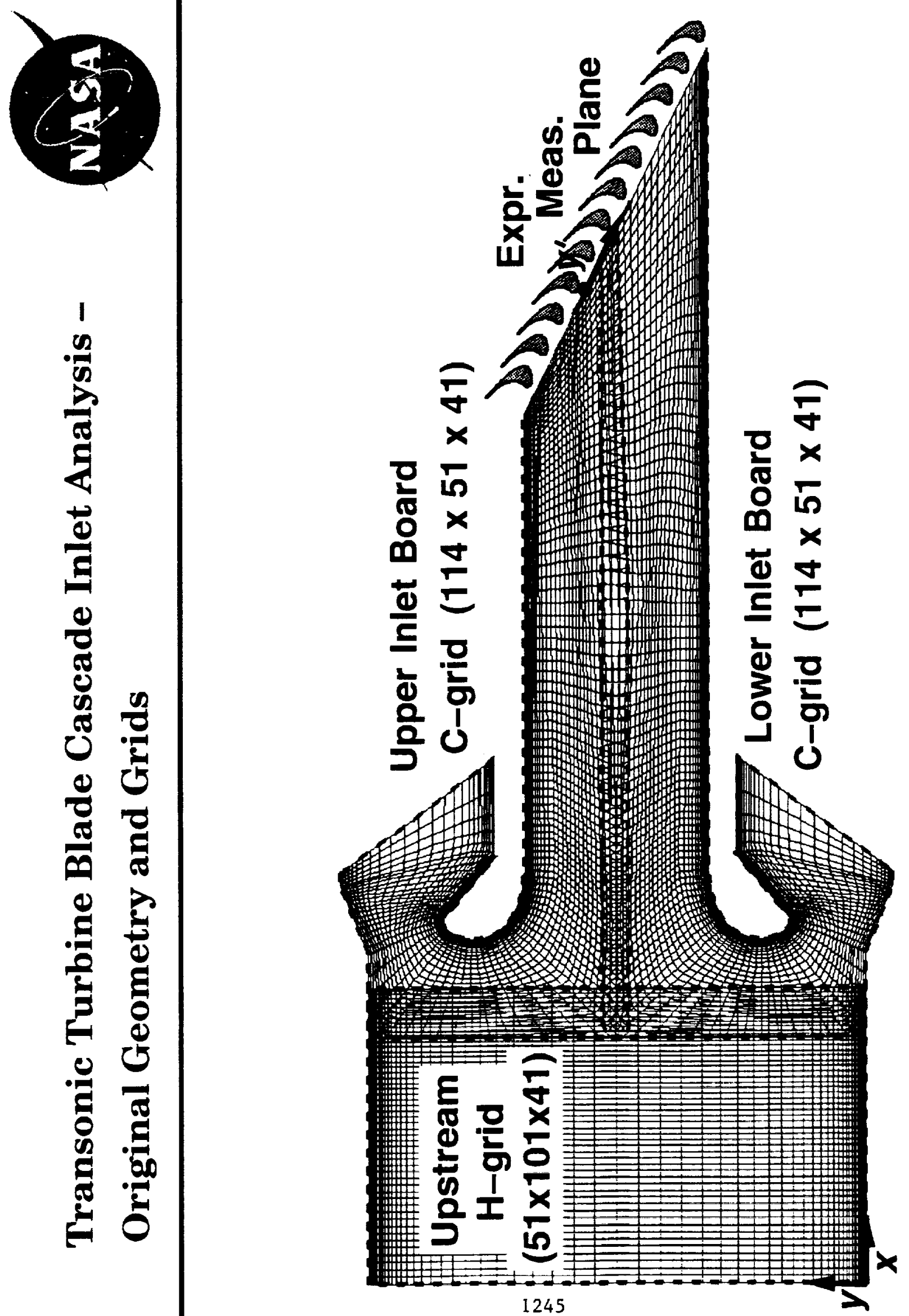


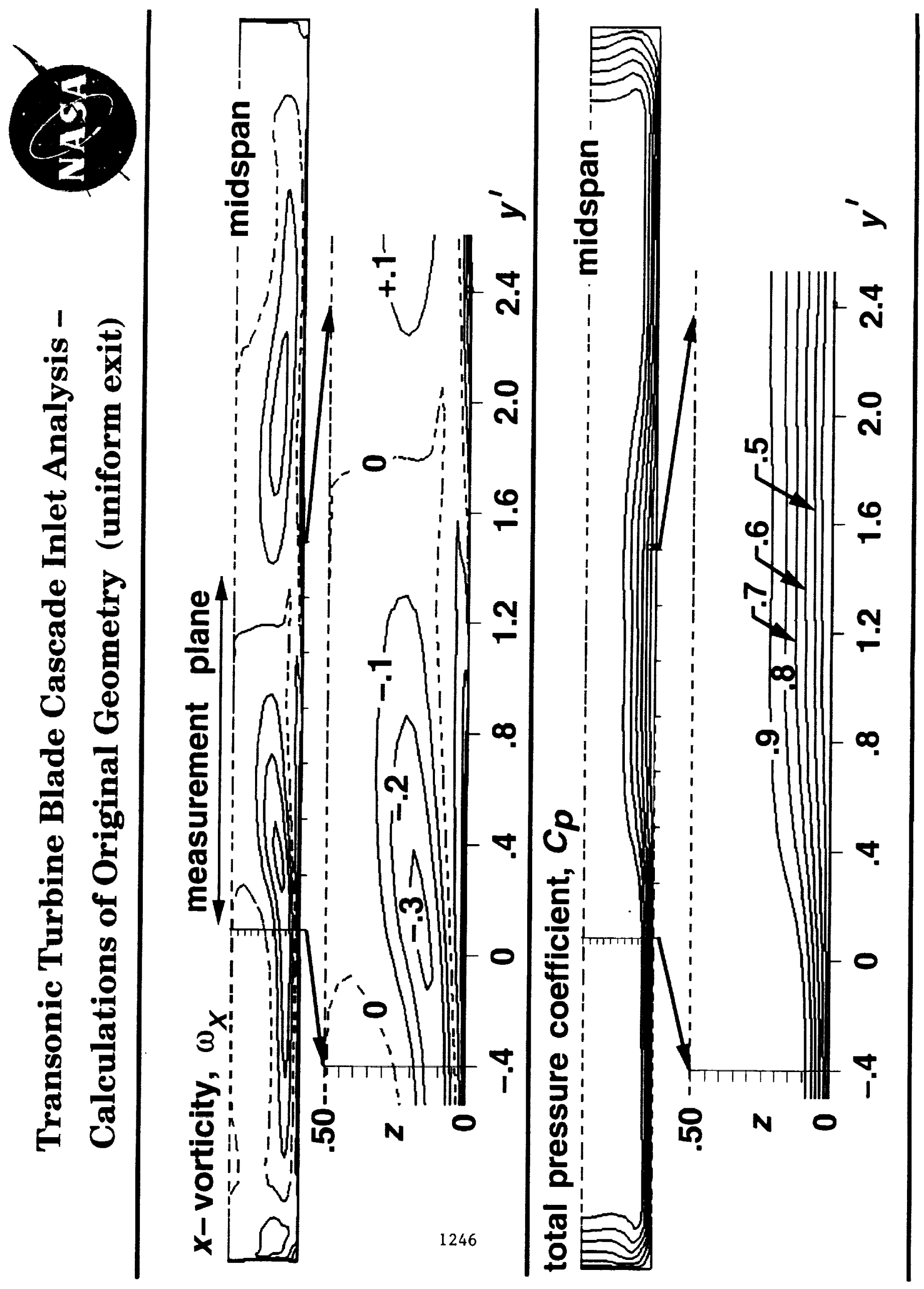



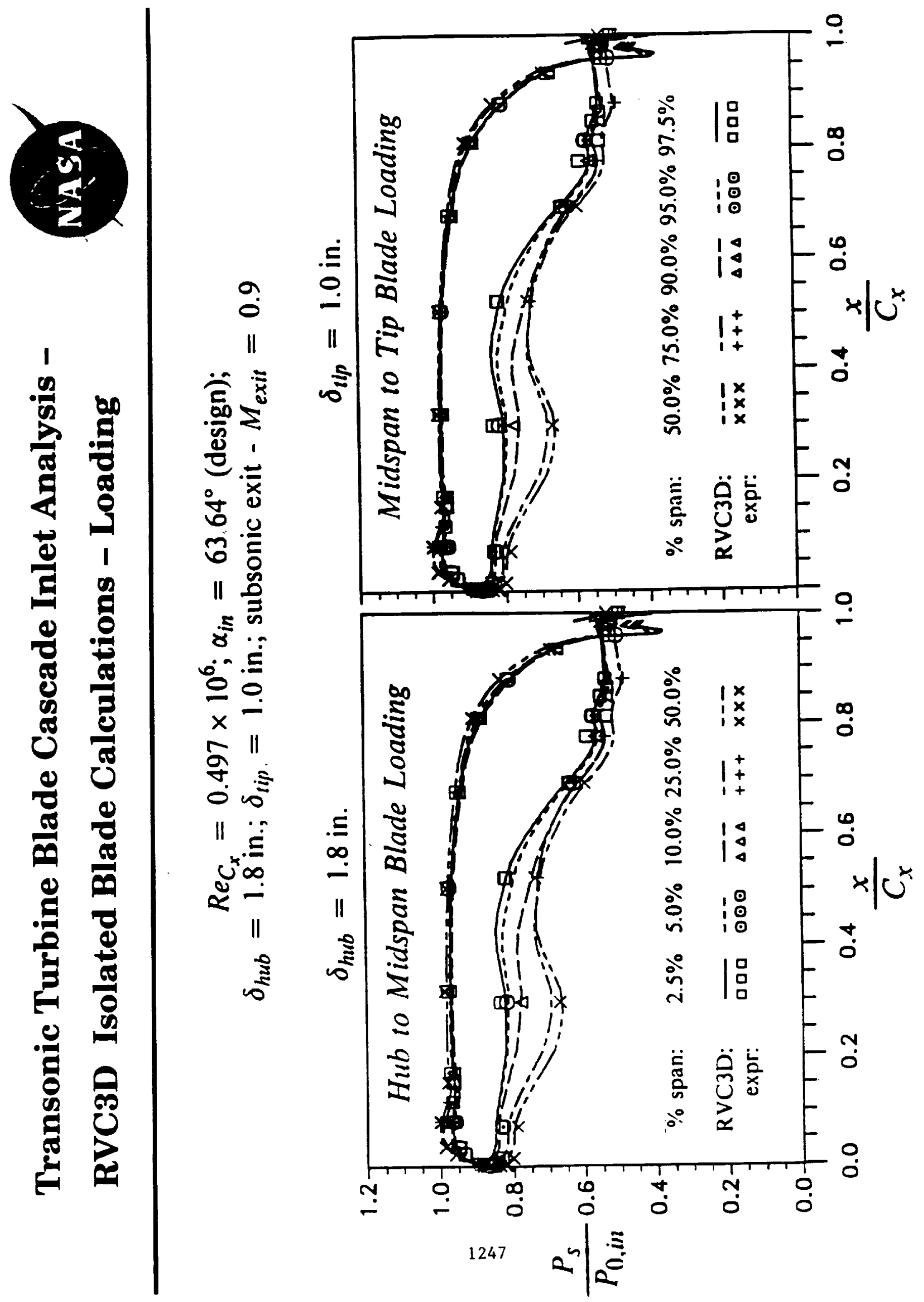


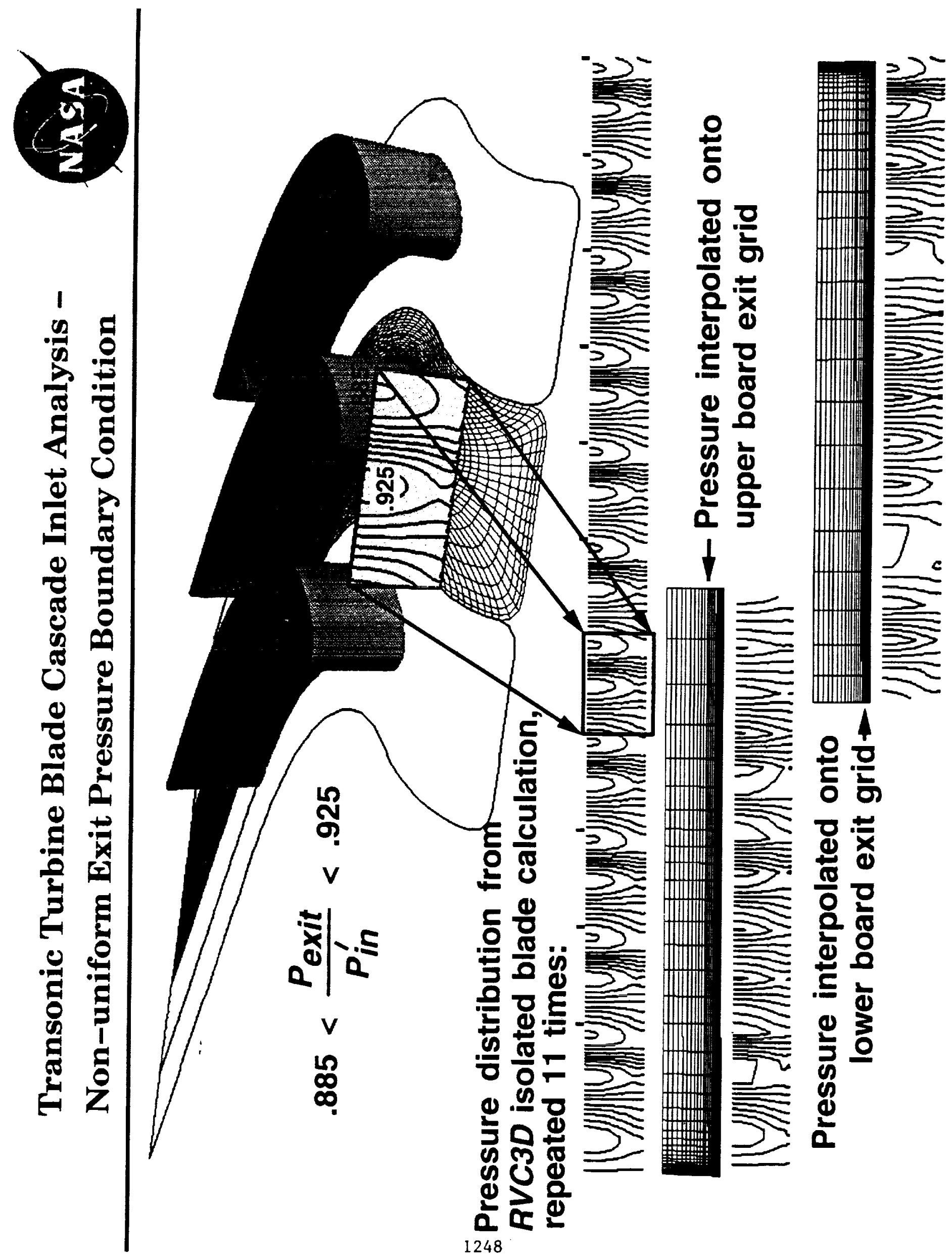



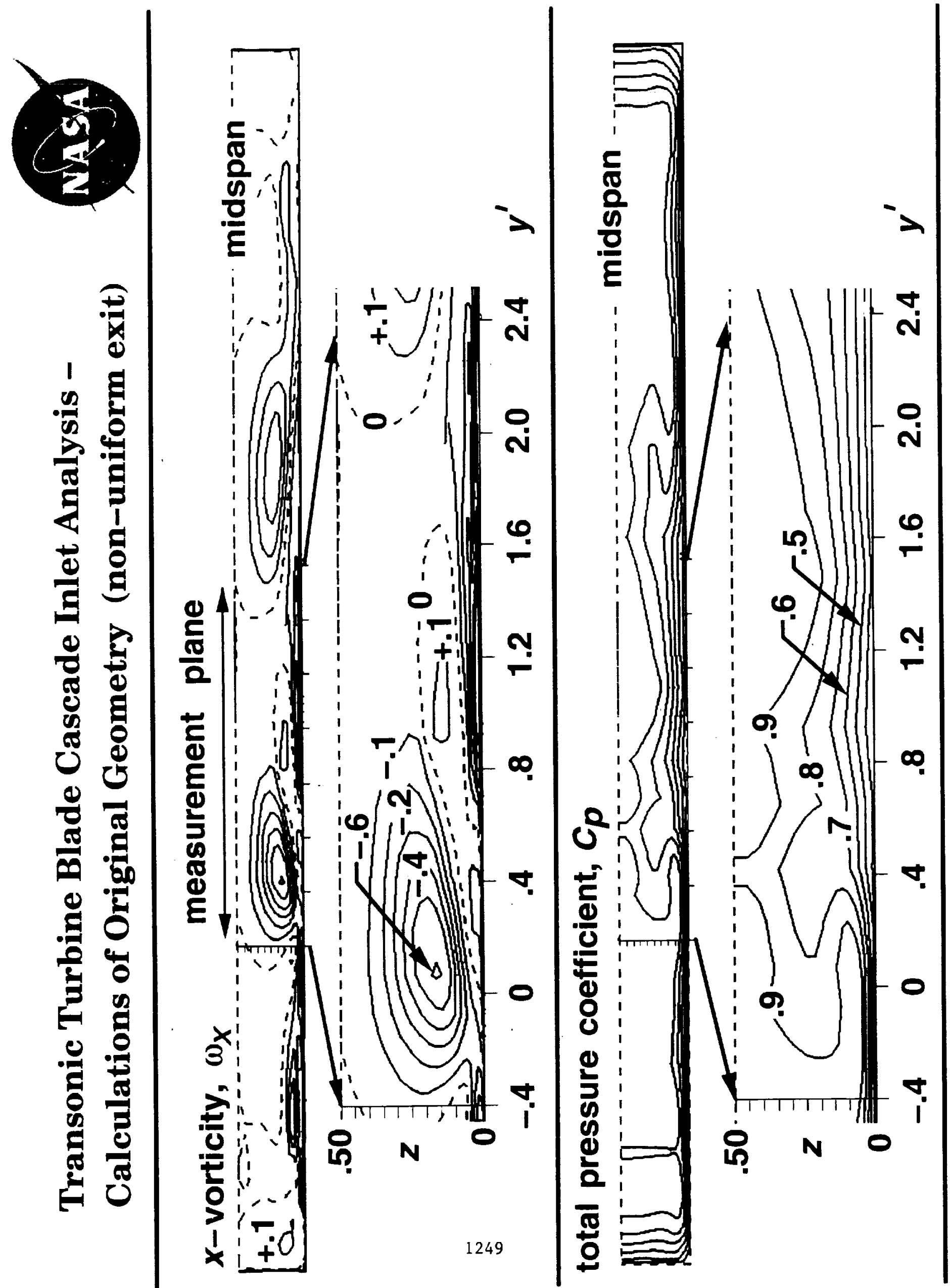

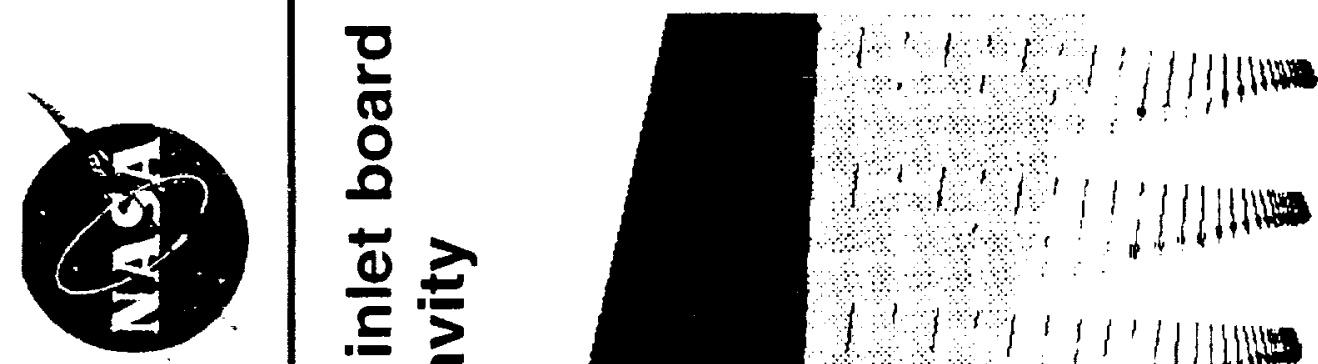

$\frac{2}{\frac{\pi}{2}}$

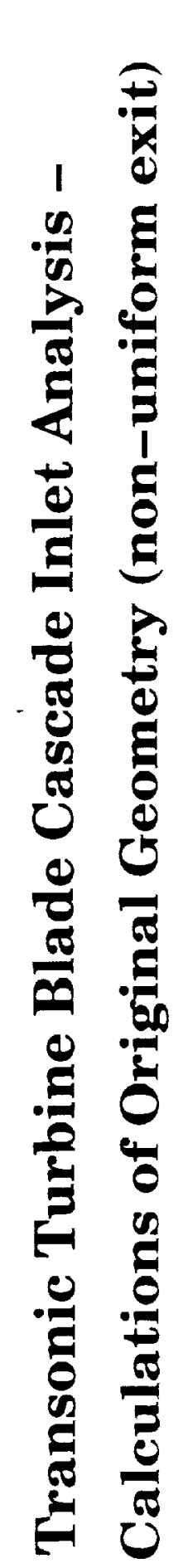



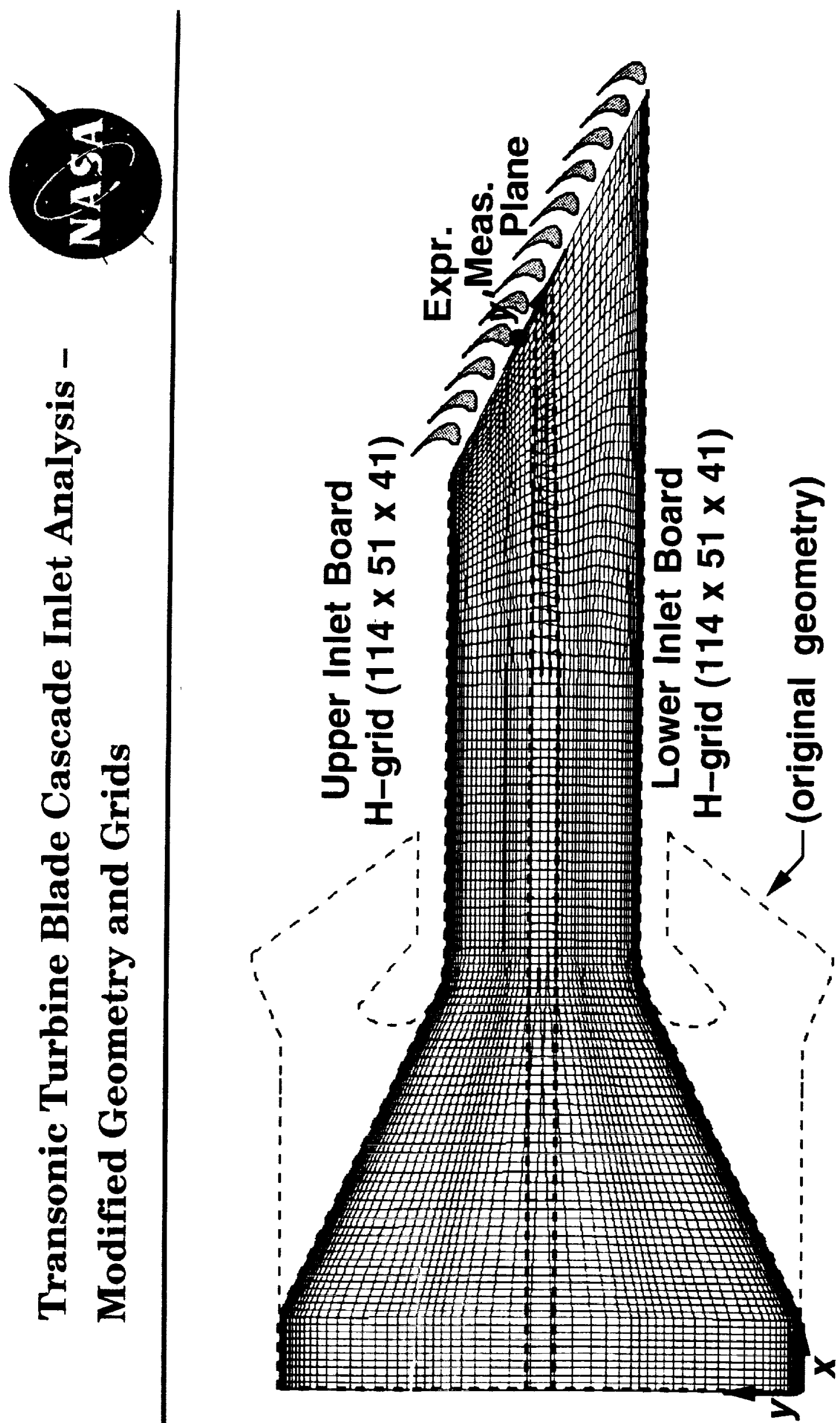

1252 
$\overparen{a}$

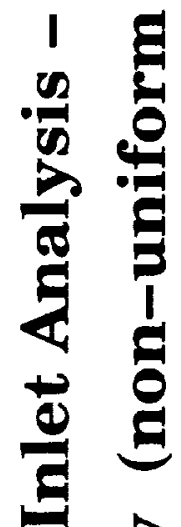

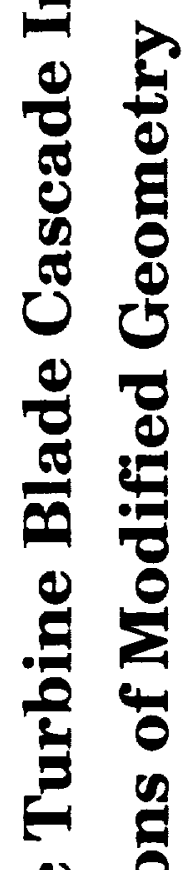

e 0

$\begin{array}{ll}8 & +5 \\ 8 & 0 \\ 6 & 0 \\ 8 & 0 \\ 8 & 0\end{array}$

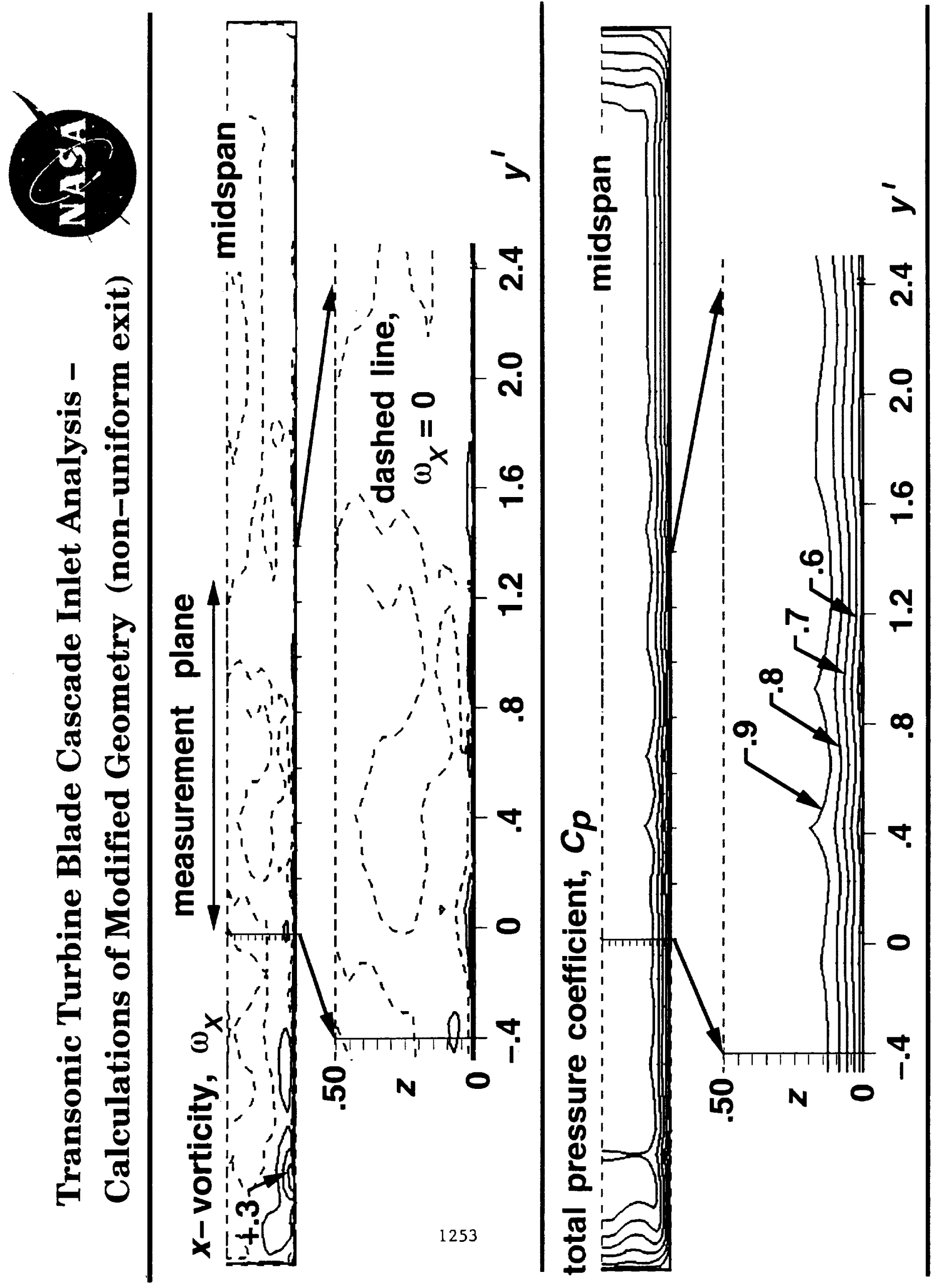

\section{.}

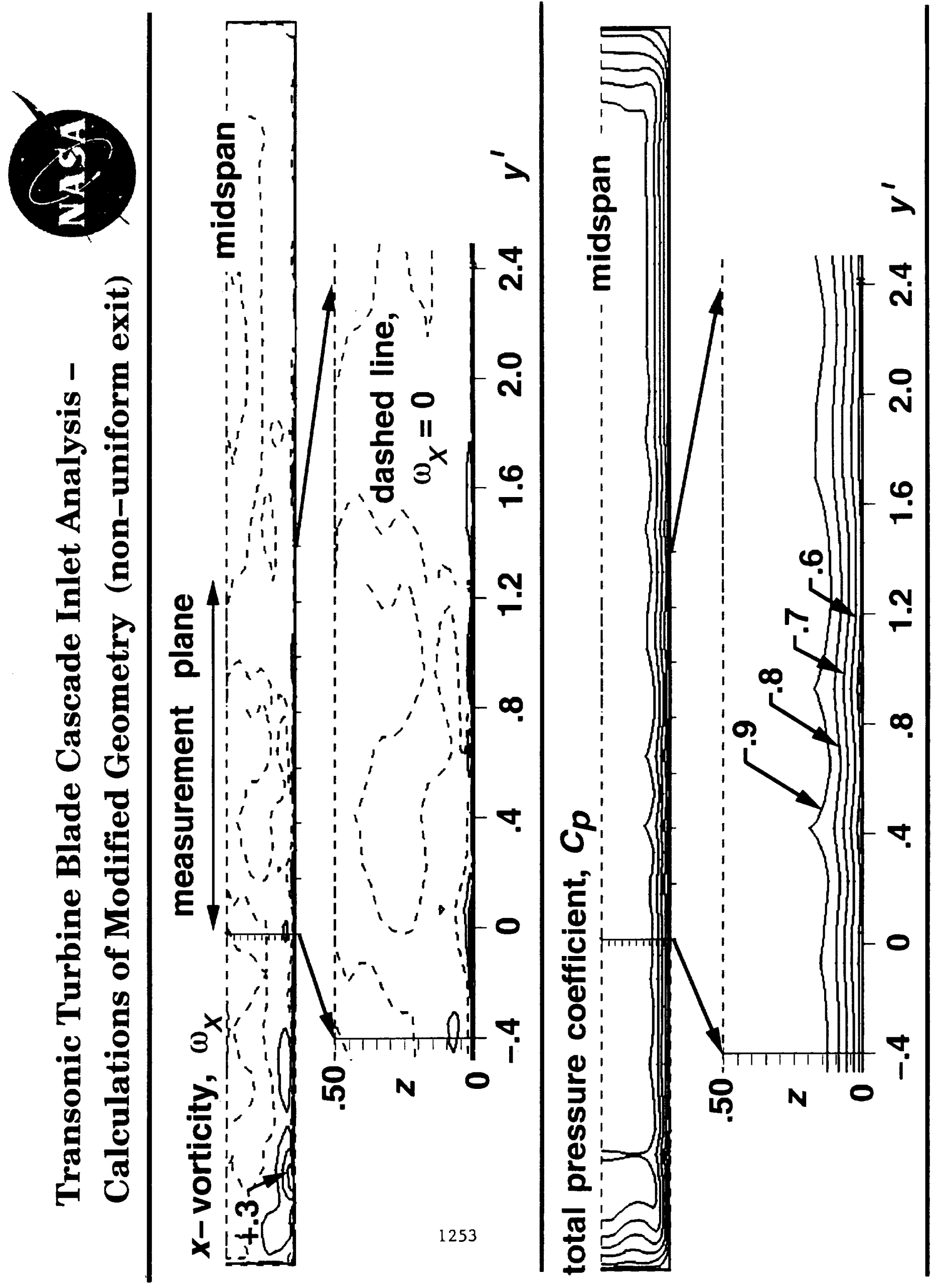



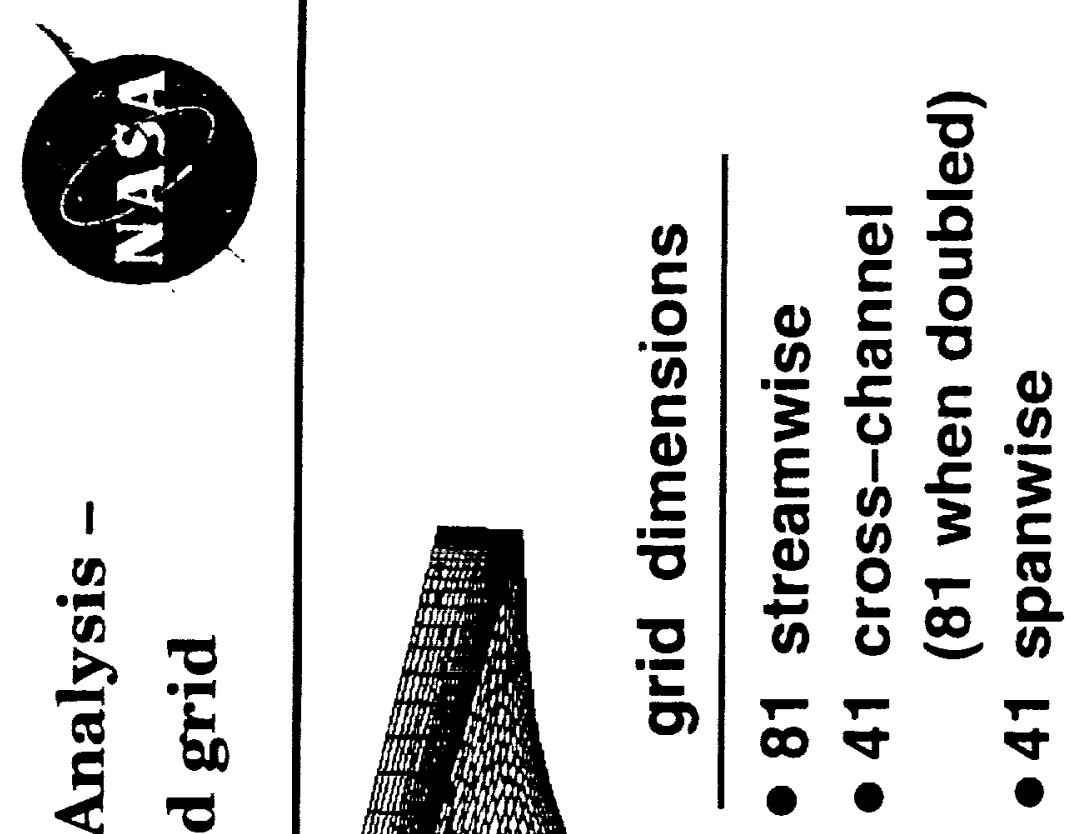


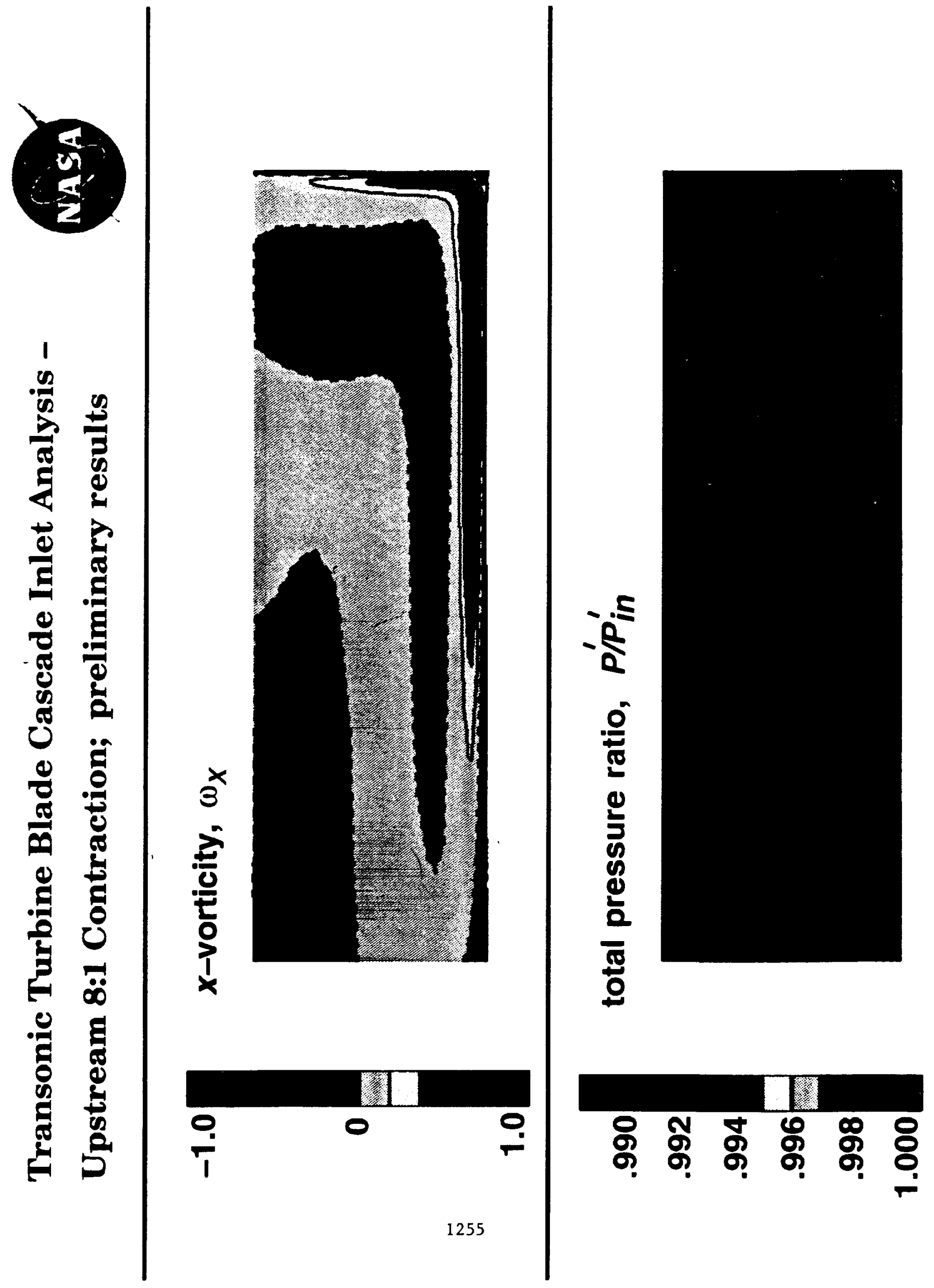




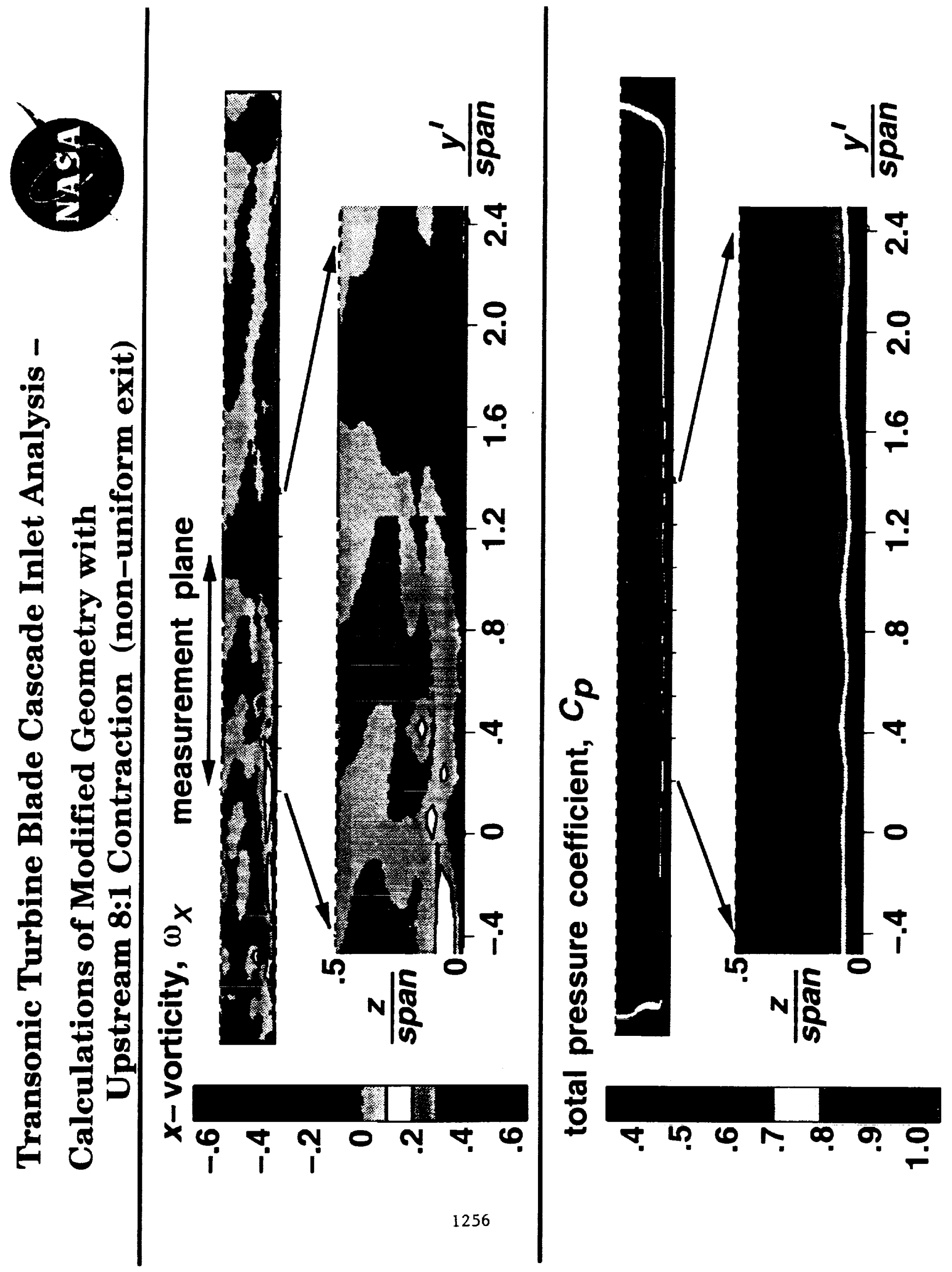



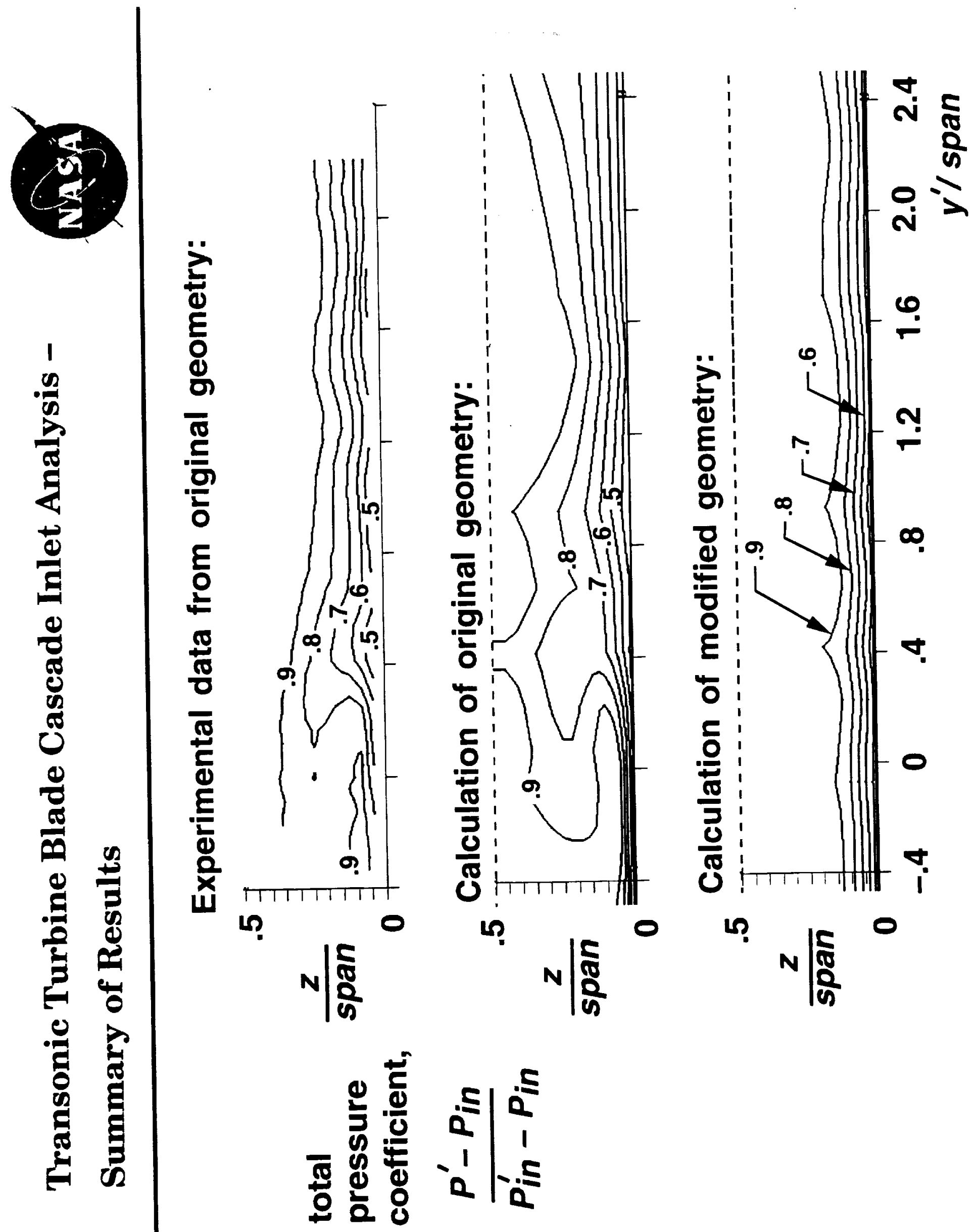


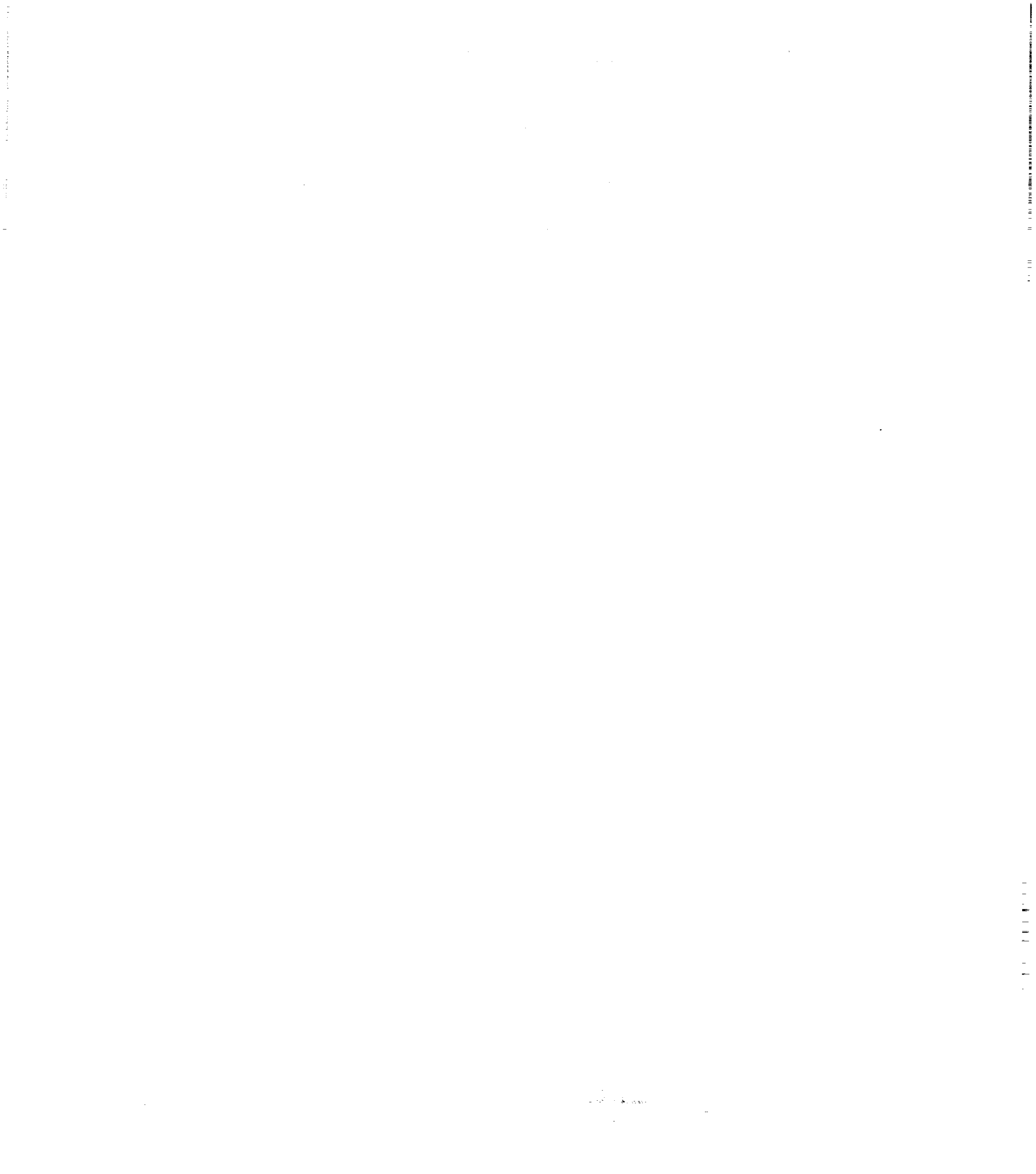

